\title{
Expectations about stimulus structure in implicit learning
}

\author{
EMMANUEL M. POTHOS \\ University of Crete, Rethymnon, Greece
}

\begin{abstract}
In two experiments, artificial grammar learning was used to create learning tasks of identical symbolic complexity that differed in terms of the instructions and the appearance of the stimuli. In Experiment 1, the stimuli were letter strings that appeared in either upper- or lowercase; however, letter case was irrelevant to the learning task. Participants were unable to take into account the instruction to ignore letter case. In Experiment 2, the stimuli were sequences of cities that corresponded to the routes of a traveling salesman. It was assumed that participants would adopt the expectation that the salesman would prefer short journeys. When the structure of the stimuli was inconsistent with this expectation, performance on the learning task was inhibited. The results suggest that there are circumstances in which explicit expectations about the structure of a set of stimuli can affect implicit learning of the stimuli.
\end{abstract}

Discussions of similarity have illustrated that a stimulus can be perceived in arbitrarily many ways (e.g., Barsalou, 1991; Goldstone, 1994; Goodman, 1972; Pothos, in press). Clearly, however, one does not blindly process each stimulus irrespective of experience; rather, expectations about its structure are used to guide perceptions. Indeed, several demonstrations have revealed how expectations about a set of stimuli can affect how the stimuli are understood. Existing research primarily concerns explicit learning of stimuli whose features can be processed independently of each other. By contrast, the present work examines how expectations about stimulus structure might affect implicit processing of stimuli that have sequential regularity.

Whittlesea and Wright (1997, p. 181) suggest that "when learning explicitly, a person encounters an object with the intention to execute some selective purpose. The intention dictates the processing performed on the object." Correspondingly, implicit learning is considered an "unselective and passive aggregation of information about the co-occurrence of environmental events and features" (Hayes \& Broadbent, 1988, p. 251). ${ }^{1}$ Explicit learning can be thought of as a collection of processing considerations about how some stimuli should be encoded. If there is some information about the structure of the stimuli, one can presumably (explicitly) modify these considerations to take into account the information. Conversely, implicit learning appears to depend on automatic processes that, by definition, normally operate independently of explicit considerations. Therefore, it is not obvious whether implicit learning could be influenced by explicit expectations about stimulus structure (cf. Destrebecqz \& Cleeremans, 2001).

I thank Todd Bailey, Nick Chater, George Sideridis, and Steven Young for their help with this project. Correspondence relating to this article should be sent to E. M. Pothos, Department of Psychology, University of Crete, Rethymnon 74100, Greece (e-mail: pothos@psy.soc.uoc.gr).
One can identify a type of knowledge that is derived from the processing of particular elements. For example, in processing an exemplar from the category cars, the actual form of each feature is of paramount importance. In such a case, each of the elements/features of a stimulus is separately meaningful and may be processed independently of the other elements (of course, processing one feature may affect the perception of the others). The influence of expectations about stimulus structure has been primarily demonstrated in the development of this form of knowledge: Presumably, expectations affect the attentional resources allocated to the different features of a stimulus (e.g., Kaplan \& Murphy, 2000). A second type of knowledge is that of sequential regularity, in which the sequential arrangement of the elements in each stimulus is of importance, not the particular format of the elements. For example, for a sequence of letters like $M S S V$ in an artificial grammar learning (AGL) experiment, the fact that the first and last symbols are different is what matters, not the actual instantiation of the symbols.

\section{Expectations About Stimulus Structure}

At a general level, Murphy and Medin (1985; Medin \& Wattenmaker, 1987) argued that categories are meaningful only if they fit our overall understanding of the world. In addition, work on psychological essentialism shows that a person often makes assumptions about a stimulus he or she encounters that go beyond its observable properties (e.g., Malt, 1994).

The assumption that a set of objects belongs to a category can induce expectations about the distributional characteristics of the objects. Markman and Hutchinson (1984) observed that the presence of a category label leads children to generalize in a taxonomic instead of a thematic way. With adult participants, Pothos, Chater, and Stewart (2004) showed that default labels will tend to be associated with objects that are highly similar to 
each other. In addition, an assumption about whether or not a set of objects belongs to linearly separable categories appears to depend on the thematic content of the objects (Wattenmaker, 1995; Wattenmaker, Dewey, Murphy, \& Medin, 1986).

Selective attentional emphasis on some stimulus properties is one of the mechanisms proposed for how expectations about a set of stimuli can affect their processing (e.g., Kruschke, 1993; Murphy \& Allopenna, 1994; Pazzani, 1991; Wisniewski, 1995). Kaplan and Murphy (2000) showed, though, that this is not quite the case. They created stimuli for which the majority of the stimulus features were designed to reflect statistical regularity (the "rote" features). At the same time, one feature in each stimulus was thematic. These thematic features were meant to increase the salience of the categorization the researchers intended for the stimuli. Kaplan and Murphy (2000) found that background information that enhanced the significance of the thematic features did not simply bias participants to focus their attention on the theme-relevant features; rather, participants appeared to learn quite a lot about the rote features as well, and in fact tried to integrate the rote features with the thematic ones (see also Kaplan \& Murphy, 1999).

The crucial aspect of the Kaplan and Murphy (2000) approach is that information about stimulus structure can affect the processing of stimuli only after the relevance of the information has been explicitly related to specific aspects of the stimuli: Participants recognize some features as theme-relevant, others as theme-irrelevant, and they modify the allocation of attentional resources accordingly. This observation applies to most of the relevant categorization studies (for an overview, see Heit, 1997). Interestingly, note that Wattenmaker (1999) showed that if there is some relevant prior knowledge for a learning task (along the above lines), the knowledge will be automatically activated, regardless of the nature of the task.

The research reviewed above does not inform us of whether expectations about the structure of a set of stimuli can affect implicit processing of sequential regularity. First, if one adopts the perspective of separate implicit and explicit learning systems, it is possible that implicit learning operates independently of any explicit considerations and biases (cf. Fodor, 1983). However, several investigators have questioned the appropriateness of the implicit/explicit distinction (e.g., Dienes \& Perner, 1999; Dulany, 2003; Tunney \& Shanks, 2003). A finding that explicit expectations about stimulus structure affect implicit learning would corroborate these concerns and possibly aid in reformulations of the implicit/explicit distinction (cf. Kinder, Shanks, Cock, \& Tunney, 2003). Second, it is not clear how expectations could influence the abstraction of sequential regularity. Presumably, expectations about stimulus structure could modulate the salience of different continuation relations among the constituent symbols of a set of stimuli, but whether such a mechanism reflects a realistic aspect of human psychology or not is an open question.

\section{Artificial Grammar Learning}

AGL experiments involve the learning of sequences of symbols usually generated from a finite state language. Finite state languages are (finite) sets of rules that specify a finite or infinite set of sequences of symbols. Each of the allowed transitions in the Figure 1 example is associated with the addition of a symbol, so that in going from the entry state to any of the exit states on the diagram, different sequences of symbols can be constructed. Sequences that are compatible or incompatible with a finite state language are called grammatical ("good"; G) or

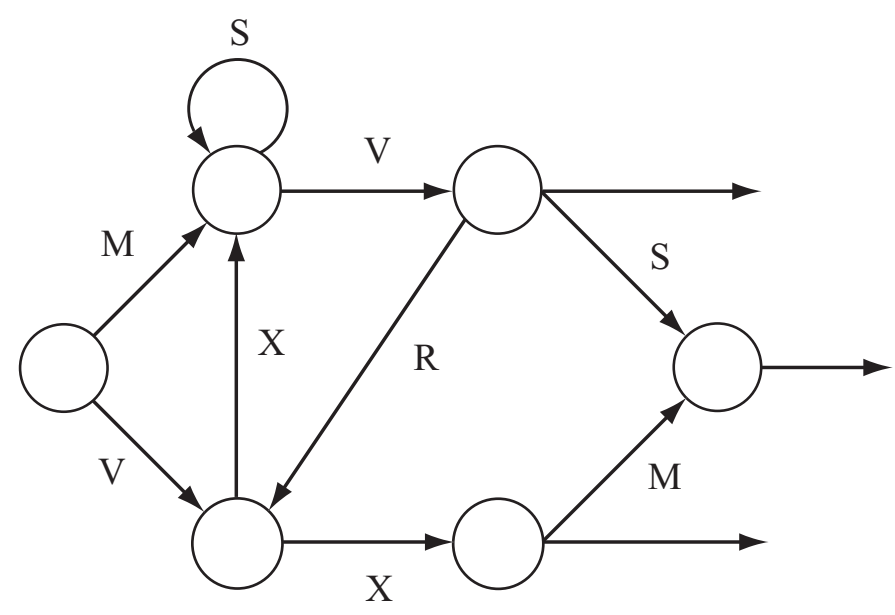

Figure 1. The finite state grammar used by A. S. Reber and Allen (1978) and the present experiments. For example, although $M S V$ would be a grammatical sequence, $V V X$ would not, since once a $V$ has appeared in a sequence, no transition could lead to the addition of a second $V$. 
ungrammatical ("not good"; NG), respectively. Note that the symbols associated with each of the allowed transitions need not be letters, but could have any form.

A. S. Reber (1967) pioneered the basic AGL paradigm. In his experiment, participants were presented with a subset of the $G$ sequences as letter strings in a training part and were asked to observe them, but no other information was provided about either the nature of the strings or what participants would do with them. Subsequently, participants were told that the strings they saw all complied with a set of rules and were asked to identify the novel $\mathrm{G}$ ones in a set that contained both $\mathrm{G}$ and $\mathrm{NG}$ strings. Participants could identify the new $G$ sequences with above chance accuracy, but they were largely unable to articulate fully the knowledge on which they based their decisions (A. S. Reber \& Lewis, 1977). A. S. Reber's (1967) study initiated a long tradition of AGL experiments in which participants consistently generalized to the $\mathrm{G}$ sequences in the test part rather than the NG ones.

AGL has been considered to reflect implicit learning, in the sense of Hayes and Broadbent (1988). Participants in an AGL task are typically clueless about how they should process the stimuli; therefore, the most readily forthcoming learning strategy appears to be passive, unselective, and unintentional processing of the information provided. In addition, all stimulus regularity is in the form of continuation relations among the constituent elements of the stimuli. AGL knowledge has been argued to correspond to rules (Dulany, Carlson, \& Dewey, 1984; A. S. Reber, 1989), similarity (Brooks \& Vokey, 1991; Pothos \& Bailey, 2000), and associative knowledge (Knowlton \& Squire, 1996; Perruchet \& Pacteau, 1990). The research reviewed in the previous section suggests that participants can explicitly direct their attention toward some features at the expense of others, depending on the corresponding stimulus expectations, when the knowledge that is developed depends on the processing of particular elements. In the case of AGL, it is not clear how stimulus expectations could translate to explicit attentional biases that would influence rule abstraction, the development of associative knowledge, or unselective sensitivity to similarity.

In sum, AGL research can further our understanding of how expectations about a set of stimuli can affect learning of the stimuli, because regularity in AGL stimuli is sequential and because AGL is generally considered to reflect implicit learning.

Finally, a controversial issue in AGL is how to assess whether learning has taken place or not. Some investigators have proposed that grammaticality accuracy of experimental participants needs to be compared with the performance of control participants who took the test without having first seen the training stimuli. Unfortunately, R. Reber and Perruchet (2003) showed that even when overall performance of control and experimental participants is the same, the basis for the G selections of the experimental participants is different from that of the controls. The presently adopted approach is to assume that if manipulations in the structure of the training phase lead to differing performance in test, then learning can be inferred. In the context of the present experiments, this reasoning turns out to be correct.

\section{EXPERIMENT 1}

An easy way to induce expectations about a set of stimuli is to tell participants to ignore some stimulus aspects. For cases in which the stimuli are made of features or dimensions that can be perceived individually, there can be little doubt that this is possible (e.g., see Nosofsky, 1991, and the references in the Expectations About Stimulus Structure section). However, as argued earlier, the situation with AGL may be different. In this experiment, standard letter-string AGL stimuli were changed so that each constituent letter of a stimulus randomly appeared in upper- or lowercase. Participants were either told that letter case was important (which should inhibit their performance) or that letter case was irrelevant. In this way, it could be established whether or not the learning process could benefit from correct expectations about the structure of the stimuli.

A. S. Reber and his colleagues explored instructional manipulations in AGL: They told participants from the outset of an AGL task that all the stimuli they were about to see were created on the basis of a set of rules, that they would have to identify these rules to perform well in the test phase to follow, and so forth. Interestingly, AGL performance deteriorated when participants were presented with these instructions, but it has been suggested that this occurred because participants simply became more anxious after receiving the instructions (Rathus, Reber, Manza, \& Kushner, 1994; A. S. Reber, Kassin, Lewis, \& Cantor, 1980; but see Shanks, Johnstone, \& Staggs, 1997).

Experiment 3 in Whittlesea and Wright's (1997) investigation involved a manipulation analogous to the one presently proposed: These investigators created an AGL task using letters in such a way that letters were never repeated back to back in the stimuli. In addition, they designed a modification of the task so that some letters in the training stimuli were randomly repeated; for example, stimulus $S M S V$ might become SMSSV. The test stimuli, however, did not contain any repetitions in either the modified or the control AGL task. Despite the fact that participants in the modified condition were told after training that repetitions were irrelevant, their performance was still significantly lower compared with that of the control participants.

This result appears to suggest that explicit expectations about stimulus structure cannot be taken into account in AGL. However, crucially, in Whittlesea and Wright's experiment participants did not know at training that repetitions were irrelevant. Therefore, one can argue that since participants processed the training items 
in terms of the spurious repetition information, they could not build up sufficient grammaticality knowledge for high performance in test. In the present experiment, expectations about stimulus structure were established at the outset of learning.

\section{Method}

Participants/Design. There were three independent sample conditions that will be referred to as same case, mixed case (case important), and mixed case (case irrelevant). Sixteen experimentally naive participants were individually tested in each condition for a small payment or course credit. The participants were students at either Oxford University or the University of Wales, Bangor.

Materials. Both experiments in this study were based on the A. S. Reber and Allen (1978) stimuli, which have been widely employed in AGL studies (e.g., Dulany et al., 1984; Perruchet \& Pacteau, 1990). Reber and Allen used the finite state language shown in Figure 1 to create 20 training items and $25 \mathrm{G}$ test items. Of the $25 \mathrm{G}$ test items, 5 were also seen in training. In addition, Reber and Allen generated $25 \mathrm{NG}$ test items by introducing specific violations to $\mathrm{G}$ items. For the mixed case conditions, I randomly converted each letter in both the training and the test AGL sequences into upper- or lowercase, so that sequences would appear as MssVRX or $V x R r$. (The G items that were repeated from training to test were converted differently for each phase.) Finally, I checked that the conversion procedure resulted in roughly equal numbers of upper- and lowercase letters.

Procedure. The general details of the procedure were based on A. S. Reber and Allen's (1978) investigation, with minor modifications. In all conditions, the training stimuli were presented twice (in Reber and Allen's study, they had been presented three times) and the test stimuli once (in Reber and Allen, twice). The appearance of each training item was identical in its two presentations in the training phase. Both training and test stimuli were shown individually at the center of a computer screen (Reber and Allen used cards). Training stimuli were shown for $10 \mathrm{sec}$. The participants saw each test stimulus until a response was provided by pressing either of two keys, labeled Good or Not Good. No feedback was provided about the correctness of the responses.

The same-case condition was the control condition. In the training phase, the participants were told that the study had two phases and that in the first they would see a series of letter strings. They were told that they would have to pay the "utmost attention to all aspects of the stimuli," but no information was provided about the test phase. After the training phase, participants learned that the "order of the letters in each of the items you just saw was determined by a rather complex set of rules" and that the "rules allow only certain letters to follow other letters." They were told that they would see another set of letter strings, half of which complied with the rules, half of which did not, and that they would have to decide which strings were which.

In the mixed case (case irrelevant) condition, the stimuli were made from mixed-case letters. The instructions provided were as above, with the following differences: In the training phase, the participants were told to pay the utmost attention to all aspects of the stimuli, but it was emphasized that "whether a letter appears in lower- or uppercase is completely irrelevant." Likewise, in the test phase, the instructions noted that case was irrelevant. By contrast, in the mixed case (case important) condition, participants were told before both training and test that whether a letter appeared in loweror uppercase was important.

\section{Results}

For each participant, I computed his or her grammaticality accuracy - that is, the proportion of $\mathrm{G}$ sequences correctly identified as $\mathrm{G}$ and the NG sequences correctly
Table 1

Mean Grammaticality Accuracy in Experiment 1

\begin{tabular}{lcc}
\hline \multicolumn{1}{c}{ Condition } & $M(\%)$ & $S D$ \\
\hline Same case & 61.87 & 7.02 \\
Mixed case (case irrelevant) & 55.12 & 5.58 \\
Mixed case (case important) & 56.12 & 7.00 \\
\hline
\end{tabular}

rejected as NG. Table 1 shows the mean grammaticality accuracy for each of the three conditions. Grammaticality accuracy was found to be significantly higher than the $50 \%$ chance performance rate in all three conditions using single-sample $t$ tests (all reported $t$ tests in this article are two-tailed) [for the same-case condition, $t(15)=$ $6.67, p<.0005$; for the mixed case (case irrelevant) condition, $t(15)=4.39, p=.001$; and for the mixed case (case important) condition, $t(15)=2.93, p=.01]$. The critical comparison focused on how performance was affected by the instructions to ignore or attend to the case of individual letters in the AGL stimuli. The result of a oneway independent-samples analysis of variance (ANOVA) comparing the means for the three conditions was significant $[F(2,45)=4.92, p=.012]$. Tukey's HSD multiplecomparisons procedure was then carried out to locate the source of significance in the ANOVA. Using an alpha level of .05 , performance in the same-case condition was found to be different from performance in the mixed case conditions; there was no difference between the mixed case conditions.

To provide a power analysis for this experiment required a conception of what kind of difference in grammaticality accuracy should be considered meaningful. Dulany et al. (1984) used the A. S. Reber and Allen (1978) training and test stimuli, and the lowest grammaticality accuracy reported in the experimental conditions was $63 \%$. Dulany et al. also ran a no-learning control; the mean grammaticality accuracy for these participants was 55\%. Similar results were reported by Altmann, Dienes, and Goode (1995; in their Experiment 1, performance for the no-learning control condition was $50 \%$, and for the experimental condition, 59\%). Therefore, at the very least, one would like to consider as significant a difference of $8 \%$ in grammaticality accuracy. Now I could evaluate whether the standard deviation and sample sizes in the conditions for which no difference was found would have been adequate to detect a difference in grammaticality accuracy of $8 \%$ or more. The pooled standard deviation for the mixed case conditions was computed to be 6.33 , so the corresponding effect size was 1.26. For $N=$ 16 , the power of identifying this effect size as significant at the .05 level was approximately $94 \%$.

\section{Discussion}

It was expected that in the mixed case (case important) condition, the participants would try to make sense of the stimuli so that upper- and lowercase versions of the same letter would be considered different symbols. In other words, participants would try to learn a different (and 
much more complex) finite state language from the one defining the G/NG distinction in the experiment. Predictably, grammaticality accuracy was very low.

Intuitively, ignoring case information seemed like a rather straightforward attentional manipulation: Participants in the case irrelevant condition should have been able to ignore case information and correctly focus on the sequential dependencies of the letters, if explicit considerations can influence implicit learning. This turned out not to be the case. This finding is consistent with Whittlesea and Wright's (1997) work, but not with the research reviewed in the section on Expectations About Stimulus Structure, in which the presence of expectations about a set of stimuli was typically associated with substantial changes in participants' performance.

An objection to this experiment (an objection also typical of AGL research) is that the learning task was contrived and entirely outside the normal experience of participants. Therefore, one could argue that the expectations about stimulus structure did not interact with the learning process in the way that realistic expectations would have affected the learning of realistic stimuli. Indeed, all of the investigators mentioned above in the Expectations About Stimulus Structure section took great care to ensure that their stimuli (and corresponding expectations) could fit into some everyday situation. Experiment 2 aimed to rectify this shortcoming.

\section{EXPERIMENT 2}

Pothos and Chater (1998) created an AGL task in a realistic setting. AGL stimuli were created as sequences of cities, such as Athens $\rightarrow$ Rome $\rightarrow$ Athens, that were meant to correspond to the locations of an airplane at noon on successive days. The city sequences were created from letter strings by a random mapping of letters to cities. Pothos and Chater found that grammaticality accuracy with the city stimuli was the same as with a corresponding AGL task using standard letter strings as stimuli. It therefore appears that participants simply interpreted the cities as arbitrary symbols.

In the present experiment, the aim was to start with city stimuli analogous to the ones used by Pothos and Chater (1998) but to create an AGL task that would induce specific expectations about the structure of the stimuli. Sequences of cities were presented as the routes a salesman had to travel. The intuitive expectation about these stimuli would be that the salesman would favor routes that linked nearby cities, so that his journeys would be as efficient as possible. Accordingly, in an intuitive version of this task, I associated high-frequency bigrams in the $\mathrm{G}$ sequences with nearby cities, whereas in the nonintuitive version the converse applied. Since the structure of the stimuli would be consistent with participants' expectations in the intuitive but not in the nonintuitive condition, if stimulus expectations can affect implicit learning one would expect higher grammaticality accuracy in the intuitive version.
Note that the above suggestion immediately raises a problem: Suppose that participants can indeed process the AGL stimuli in terms of whether or not they are consistent with the intuition that the salesman makes many short trips. In the intuitive version, the $\mathrm{G}$ sequences in test would be differentiated from the NG ones both because they are legal and because they are associated with shorter journeys. Therefore, participants might be discriminating between $\mathrm{G}$ and $\mathrm{NG}$ sequences in test not on the basis of any knowledge acquired during training, but rather by using their intuition about the salesman making several short trips in order to be profitable. Accordingly, to establish whether learning is taking place, a no-learning control condition was included in this experiment.

\section{Method}

Participants/Design. The experiment involved three independentsamples conditions: In the intuitive condition, high-frequency bigrams generally corresponded to nearby cities; in the nonintuitive condition, the converse applied; finally, in the control condition, the correspondence of cities with letters was the same as in the intuitive condition, but participants went through the test items without having first seen the training items. Ten experimentally naive participants were tested in each condition for a small payment or course credit. The participants were students at either Oxford University or the University of Wales, Bangor.

Materials. The materials were again based on the A. S. Reber and Allen (1978) letter strings. In order to derive a correspondence between cities and letters that would be consistent with the motivation for the intuitive or the nonintuitive condition, I first counted the number of bigrams (i.e., pairs of letters appearing consecutively) in the training items and the test items (this information is shown in Appendix A; in all appendixes, the bigrams or AGL stimuli are specified in terms of the letters shown in Figure 1). Note that the (Pearson) correlation between the frequencies of the common bigrams for test $\mathrm{G}$ items and training items was .75 , but the corresponding correlation for the NG items was .61. I next generated a matrix for all possible bigrams in training; each cell of the matrix would correspond to a bigram frequency. Hence, the entry for $M S$ would be 5 (see Appendix A), and so forth. If $x y$ was a cell of this matrix whose value could be specified by the frequency of the bigram in the training items, yet for $y x$ this was not possible, the value for $y x$ was set to be the same as that for $x y$. Also, when there were different frequencies for $x y$ and $y x$, the higher frequency was used as the value of both cells. For these reasons, the resulting matrix was only partly consistent with the structure of the AGL stimuli (e.g., $R X$ was a highly frequent bigram in both training and the test $\mathrm{G}$ items, but $X R$ violated the Figure 1 sequential constraints). Finally, neither $S R$ nor $R S$ appeared in training nor in the test $\mathrm{G}$ items, and the values of their corresponding cells were set to 1 .

I then subtracted the value of each cell from 11 (the highest bigram frequency was 10), since I wanted to associate high bigram frequencies with short distances, and carried out a multidimensional scaling procedure (MDS; Shepard, 1980) on the modified matrix. This procedure produced the Figure 2 arrangement of letters (the stress of the MDS solution was only .013, indicating a very good fit between the modified matrix and the spatial arrangement of the letters; the two dimensions were arbitrary). To reiterate, this spatial arrangement of letters has been derived with a view of associating pairs of letters corresponding to higher frequency bigrams (modulo the simplifications noted in the previous paragraph) with shorter distances. By translating $S$ to Norwich, $M$ to Birmingham, $X$ to Leeds, $V$ to Sheffield, and $R$ to Manchester, it is possible to map the MDS solution to realistic U.K. geography (Figure 3). All of the stimuli are well-known British cities, and the assumption was 


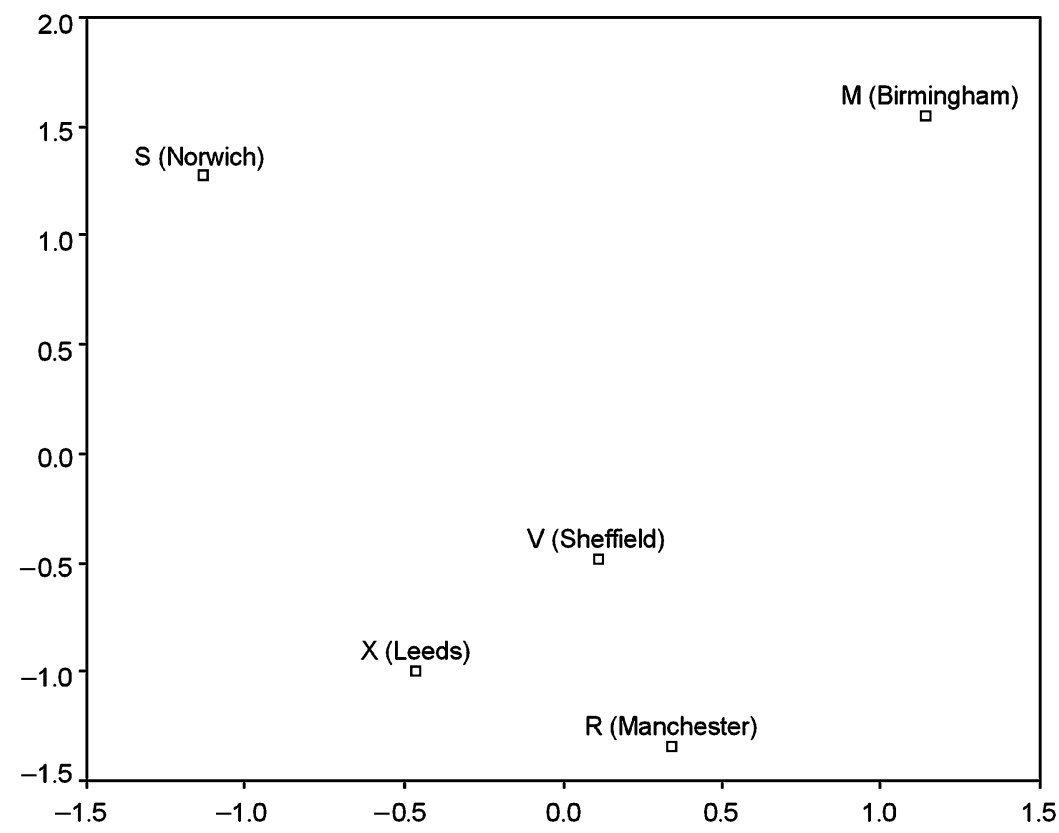

Figure 2. A spatial arrangement of the letters used in the A. S. Reber and Allen (1978) stimuli in which letters closer together correspond to higher frequency bigrams in the training stimuli. A correspondence of letters to cities can readily be established to reflect realistic U.K. geography (Figure 3).

that students at British universities (the participants in the experiment) would be familiar with them. In the Results section, I discuss whether or not the results indicate this assumption to have been accurate. This association of letters with cities corresponds to the intuitive condition in this experiment.

I next consider whether the MDS solution indeed reflects a correspondence between high-frequency bigrams and shorter distances. Intuitively, the highest frequency bigrams in training, $V X(10), V R$ (10), $R X(10)$, and $S V(9)$, are all associated in the mapping with short distances, except for one $(S V)$. Moreover, the $R S / S R$ bigrams that do not appear in training and other low-frequency bigrams$X S$ (4), $X M(2)$, and $R M(1)$ - all correspond to longer distances. A more specific analysis for this issue has been provided by assigning a 0,1 , or 2 value to each bigram, depending on whether it reflects a short, medium, or long distance in Figure 2 (see Appendix B). Clearly, classifying distances in this way involves considerable approximation; however, it is unlikely that the participants' naive knowledge of the distances between U.K. cities would be any more specific. I then computed the overall distance corresponding to each AGL test stimulus. For example, bigrams $M S$ and $S V$ were associated with a distance of 1 , and $V V$ with a distance of 0 . Therefore, string $M S V V$ would correspond to an overall distance of $1(M S)$ plus $1(S V)$ plus $0(V V)$, for a total of 2 . Appendix $\mathrm{C}$ shows the total distance corresponding to each of the test items in A. S. Reber and Allen's (1978) AGL task. The average distance of the test $\mathrm{G}$ items is $1.60(S D=1.04)$, and of the NG items, $2.32(S D=1.63)$. In conclusion, it can be seen that in the intuitive condition of this experiment, the expectation that the salesman should organize his trips so that he visits nearby cities as frequently as possible is reflected in the structure of the training stimuli; on average, the G sequences in test correspond to shorter distances than the NG ones.

For the nonintuitive condition, I simply changed the association between letters and cities so that high-frequency bigrams no longer corresponded to short distances. Hence, $S$ was mapped to Leeds, $M$ to Sheffield, $X$ to Manchester, $V$ to Norwich, and $R$ to Birmingham.
With these new associations, the $V X R$ triangle, which is the result of the high-frequency bigrams in the training items, now corresponds to cities as far apart as possible in Figure 2.

Given the above associations between letters and cities for the intuitive and nonintuitive conditions, the stimuli were created in a straightforward way by replacing the letters in the A. S. Reber and Allen (1978) stimuli with cities. Therefore, for example, in the nonintuitive condition the string $M S M$ would appear as Sheffield $\rightarrow$ Leeds $\rightarrow$ Sheffield. Two sets of training and test stimuli were created, one for the intuitive condition and the other for the nonintuitive one.

Procedure. In the intuitive and nonintuitive conditions, the 20 distinct training stimuli were presented individually three times at the center of a computer screen; each stimulus was shown until the space bar was pressed. No stimulus was presented $n+1$ times before all stimuli had been presented $n$ times. In all three conditions, the $25 \mathrm{G}$ and $25 \mathrm{NG}$ items were presented individually at the center of a computer screen until a response was made; each test item was shown twice. The participants indicated a response by pressing keys labeled Good or Not Good; no corrective feedback was provided.

For the training phase of the intuitive condition, participants were told that they were about to see different routes used by a traveling salesman and that city names corresponded to places the salesman would have to visit on successive days (repeated city names would indicate that the salesman made a visit to a place near the main city or that he took the day off). It was then noted that "The routes of the salesman were planned so as to maximize travel efficiency: The secret to success is as many trips between nearby cities as possible. Long trips are not so efficient; the salesman has to wait more time before making contact with customers." After the training phase, the participants were informed that "The salesman has figured out a set of rules to plan his trips; the routes allowed by these rules are generally efficient and the salesman has used them for a long time." They were then told that all the routes in the first phase complied with these rules, that they were about to see new routes either consistent or not consistent with the rules, and that their task was to 


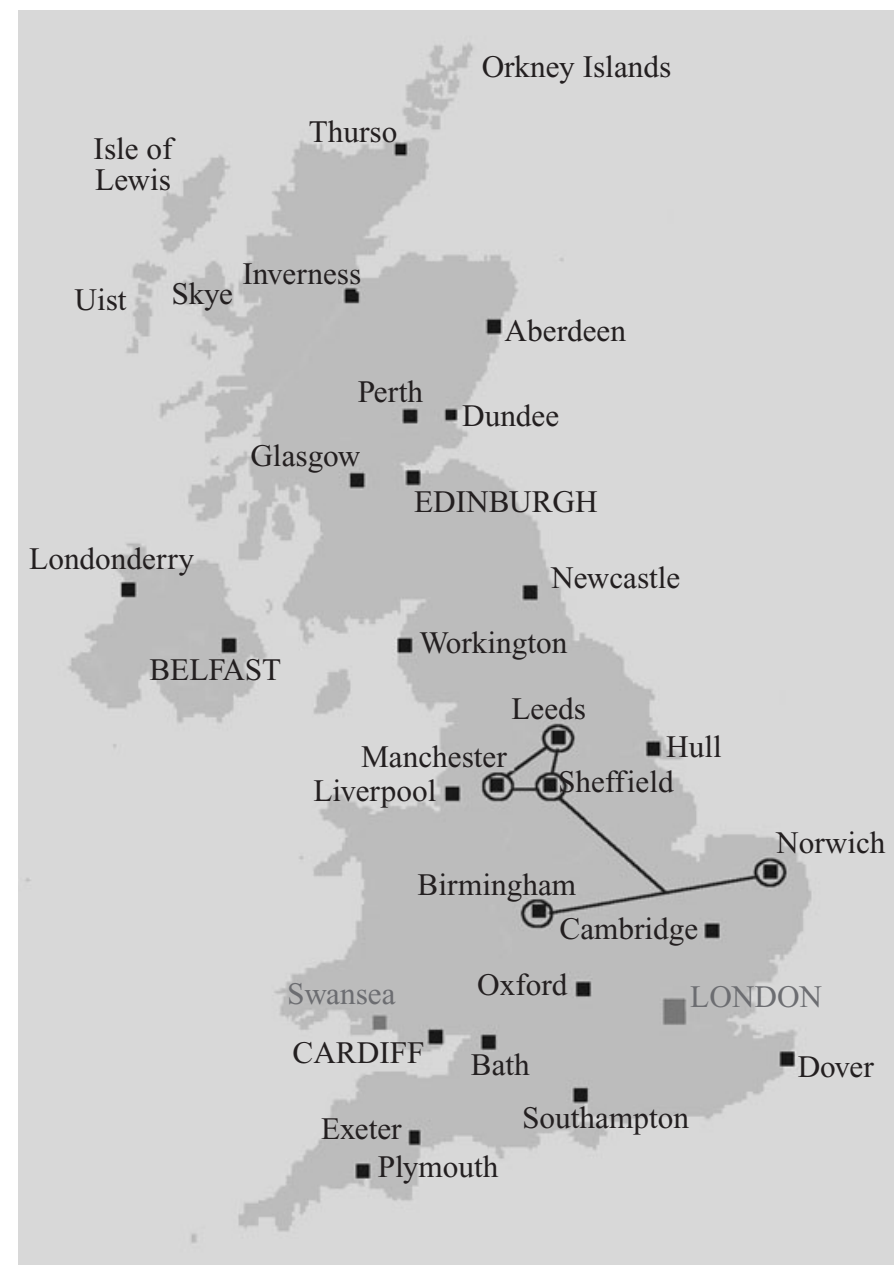

Figure 3. A map of the U.K. in which the particular correspondences between letters and cities in Figure 2 are justified.

discriminate between the two. The instructions and procedure for the nonintuitive condition were identical to those for the intuitive condition; the two conditions differed only with respect to the stimuli used.

In the control condition, the participants were asked to discriminate between the G and NG stimuli (corresponding to the intuitive condition) without first having seen the training stimuli. They were given the same story about the traveling salesman and how he had a set of rules to decide which routes were advantageous and which were not. The participants were then told that some of the routes they were about to see were consistent with these rules and that some were not, and that they would have to distinguish between the two.

\section{Results}

As in Experiment 1, the analyses were based on grammaticality accuracy; the average performance and standard deviation for each of the three conditions are shown in Table 2. In this experiment, grammaticality accuracy was found to be higher than the $50 \%$ chance level in the intuitive and control conditions $[t(9)=4.31, p=.002$, for the intuitive condition; $t(9)=2.73, p=.023$, for the control condition]. For the nonintuitive condition, the re- sult was $t(9)=1.64, p=.137$. The experimental hypothesis related to whether performance in the intuitive condition was different from performance in the nonintuitive one, and this turned out to be the case, as assessed by an independent-samples $t$ test $[t(18)=2.734, p=$ .014]. The means of the three conditions were compared in a one-way independent-samples ANOVA, which was found significant $[F(2,27)=5.38, p=.011]$. Furthermore, Tukey's HSD procedure (alpha $=.05$ as before) showed that the only significant differences were between the intuitive and the other two conditions.

I then repeated the power analysis outlined in Experiment 1 . The pooled standard deviation for the conditions

Table 2 Mean Grammaticality Accuracy in Experiment 2

\begin{tabular}{lcc}
\hline \multicolumn{1}{c}{ Condition } & $M(\%)$ & $S D$ \\
\hline Intuitive & 60.20 & 7.48 \\
Nonintuitive & 52.50 & 4.84 \\
Control (no learning) & 53.60 & 4.17 \\
\hline
\end{tabular}


in which there was no difference was 4.52 , so the effect size for a grammaticality difference of $8 \%$ would be 1.77 . For $N=10$, the power of identifying this effect size as significant at the .05 level was approximately $97 \%$.

\section{Discussion}

Grammaticality accuracy in the control condition was in line with the accuracy reported for the no-learning controls of Dulany et al. (1984) and Altmann et al. (1995). In other words, for control participants, apparently the intuition that the salesman should prefer short trips is not sufficient to guide performance in the absence of corresponding knowledge from processing stimulus structure, even though in principle this intuition could have led to high discrimination accuracy between $\mathrm{G}$ and NG sequences.

Performance in the intuitive condition $(60.20 \%)$ was nearly identical to that in the same-case condition (61.87\%) of Experiment 1. This result appears to show that when the expectations about stimulus structure were consistent with the actual structure of the stimuli, learning proceeded more or less as it would have done in the absence of any expectations. This conclusion is not necessarily correct, however, for the following reason: The grammaticality accuracy measure with the A. S. Reber and Allen (1978) stimuli has been shown to reflect a variety of knowledge influences, such as rules knowledge (which was the original intention of Reber and Allen), similarity (e.g., Brooks \& Vokey, 1991), or distributional fragment knowledge (e.g., Perruchet \& Pacteau, 1990). Therefore, it is possible that for example, a different mix of rules and similarity influences led to the $60.20 \%$ accuracy in the intuitive condition than to the $61.87 \%$ of the same-case condition (cf. Pothos \& Bailey, 2000). Unfortunately, the Reber and Allen materials that were used are not well balanced, so the issue just raised cannot be explored presently.

In the nonintuitive condition, grammaticality performance was indistinguishable from that of the no-learning controls. In this case, there would be a conflict between the knowledge acquired by processing the statistical structure of the stimuli and the explicit expectations about stimulus structure. Apparently, this conflict prevented learning. If one assumes that AGL reflects implicit learning, this result is a demonstration of explicit expectations affecting implicit learning of a set of stimuli.

The assumptions in this experiment were numerous: First, I assumed that participants would be familiar with the relevant British geography; second, that participants would consider common sense and adopt the expectation that the salesman would prefer many short trips; third, that this expectation, together with their knowledge of British geography, would make certain city sequences in the AGL stimuli more plausible than others; and fourth, that the implicit learning process could be influenced by such a sense of plausibility and affect the encoding of the AGL stimuli. That these assumptions were in fact accurate, however, is the most straightforward interpretation of the result that grammaticality accuracy was much lower in the nonintuitive than in the intuitive condition.
If the assumptions were invalid (e.g., because participants knew nothing of British geography), then there would be no basis for a performance difference between the intuitive and nonintuitive conditions - sequences of cities would be processed as sequences of symbols, without regard to their meaning (cf. Pothos \& Chater, 1998).

\section{GENERAL DISCUSSION}

In Experiment 1, expectations about the structure of the stimuli were established in a seemingly straightforward way, by telling participants to ignore or attend to certain aspects of the stimuli. In the critical condition, participants were apparently unable to ignore letter case and to perform as well as those responding to the standard letter string stimuli. In Experiment 2, a possible expectation about the structure of the stimuli was established in a more complex way. It was assumed that participants would adopt the (instructional) suggestion that the salesman would prefer several short trips and that therefore, using some basic knowledge of British geography, participants would be able to identify certain sequences of cities as more plausible than others. The results of the experiment suggest that high accuracy of discrimination between the $\mathrm{G}$ and NG sequences could not be guided solely by an overall expectation about long versus short trips. Rather, a synergy was required between the stimulus structure and the corresponding explicit expectations.

It is puzzling that the results failed to reveal an influence of expectations in the seemingly straightforward case of Experiment 1, and yet did so in the much more complex case of Experiment 2. However, a possible account for this pattern of results is the following: In Experiment 2, the basis of the knowledge acquired would presumably correspond, to a large extent, to bigram information (cf. Perruchet \& Pacteau, 1990). Expectations about stimulus structure were established in terms of such information, so that apparently they could directly interface with and modulate the learning mechanism processing stimulus structure. By contrast, that was not the case in Experiment 1, in which expectations about the stimuli referred to an aspect of the stimuli that had to be ignored. Apparently, participants could not both ignore letter case and process the sequential dependencies of the stimuli (cf. the Stroop paradigm or negative priming: MacLeod, 1991; Tipper \& Cranston, 1985). This possibility could be further explored by manipulating bigram frequencies and corresponding expectations in more controlled ways and by examining whether the salience of certain bigrams can indeed be enhanced when they are consistent with the corresponding expectations.

The present results do not support the view of an implicit learning mechanism that is impenetrable to explicit considerations about the stimuli that are processed. Ideally, to clarify this issue further, a modification of the basic AGL paradigm would be needed whereby participants are examined on how they make their grammaticality decisions in test, as they go through the test phase 
(cf. Dulany et al., 1984). This is an issue for further work, however.

The most important limitation of the present study is that it assessed AGL performance only in terms of grammaticality accuracy. As discussed in the Results section of Experiment 2, the same grammaticality accuracy could reflect several different kinds of knowledge. In addition, several investigators have demonstrated that test item classification is quite different when participants are asked to identify "legal" sequences (a direct measure of performance) and when they are asked which sequences they like or prefer (an indirect measure of performance; see Helman \& Berry, 2003; Higham, Vokey, \& Pritchard, 2000; Newell \& Bright, 2001). Direct and indirect measures of performance plausibly address different aspects of the learning outcome. It is therefore likely that if the present investigation were to be extended to include indirect measures of performance, the conclusions derived would likewise be different.

Despite the above qualifications, the present experiments do demonstrate some conditions under which expectations about stimulus structure do or do not affect performance in AGL and offer some perspective toward directions for future research.

\section{REFERENCES}

Altmann, G. T. M., Dienes, Z., \& Goode, A. (1995). Modality independence of implicitly learned grammatical knowledge. Journal of Experimental Psychology: Learning, Memory, \& Cognition, 21, 899-912.

BARSALOU, L. W. (1991). Deriving categories to achieve goals. In G. H. Bower (Ed.), The psychology of learning and motivation (Vol. 27, pp. 1-64). New York: Academic Press.

Brooks, L. R., \& VoKey, J. R. (1991). Abstract analogies and abstracted grammars: Comments on Reber (1989) and Mathews et al. (1989). Journal of Experimental Psychology: General, 120, 316-323.

Destrebecqz, A., \& Cleeremans, A. (2001). Can sequence learning be implicit? New evidence with the process dissociation procedure. Psychonomic Bulletin \& Review, 8, 343-350.

Dienes, Z., \& Perner, J. (1999). $\bar{A}$ theory of implicit and explicit knowledge. Behavioral \& Brain Sciences, 22, 735-808.

Dulany, D. E. (2003). Strategies for putting consciousness in its place. Journal of Consciousness Studies, 10, 33-43.

Dulany, D. E., Carlson, R. A., \& Dewey, G. I. (1984). A case of syntactical learning and judgment: How conscious and how abstract? Journal of Experimental Psychology: General, 113, 541-555.

FoDOR, J. A. (1983). The modularity of mind. Cambridge, MA: MIT Press.

Goldstone, R. L. (1994). The role of similarity in categorization: Providing a groundwork. Cognition, 52, 125-157.

Goodman, N. (1972). Problems and projects. Indianapolis: BobbsMerrill.

Hayes, N. A., \& Broadbent, D. E. (1988). Two modes of learning for interactive tasks. Cognition, 28, 249-276.

Heit, E. (1997). Knowledge and concept learning. In K. Lamberts \& D. Shanks (Eds.), Knowledge, concepts, and categories (pp. 7-41). Hove, U.K.: Psychology Press.

Helman, S., \& Berry, D. C. (2003). Effects of divided attention and speeded responding on implicit and explicit retrieval of artificial grammar knowledge. Memory \& Cognition, 31, 703-714.

Higham, P. A., Vokey, J. R., \& Pritchard, J. L. (2000). Beyond dissociation logic: Evidence for controlled and automatic influences in artificial grammar learning. Journal of Experimental Psychology: General, 129, 457-470.

KAPLAN, A. S., \& MurPhy, G. L. (1999). The acquisition of category structure in unsupervised learning. Memory \& Cognition, 27, 699712.

Kaplan, A. S., \& Murphy, G. L. (2000). Category learning with minimal prior knowledge. Journal of Experimental Psychology: Learning, Memory, \& Cognition, 26, 829-846.

KINDER, A., Shanks, D. R., COCK, J., \& Tunney, R. J. (2003). Recollection, fluency, and the explicit/implicit distinction in artificial grammar learning. Journal of Experimental Psychology: General, 132, 551-565.

KNOWLTON, B. J., \& SQuiRe, L. R. (1996). Artificial grammar learning depends on implicit acquisition of both abstract and exemplar-specific information. Journal of Experimental Psychology: Learning, Memory, \& Cognition, 22, 169-181.

KRUSCHKE, J. K. (1993). Three principles for models of category learning. In G. V. Nakamura, R. Taraban, \& D. L. Medin (Eds.), Categorization by humans and machines: The psychology of learning and motivation (Vol. 29, pp. 57-90). San Diego: Academic Press.

MacLeod, C. M. (1991). Half a century of research on the Stroop effect: An integrative review. Psychological Bulletin, 109, 163-203.

Malt, B. C. (1994). Water is not $\mathrm{H}_{2} \mathrm{O}$. Cognitive Psychology, 27, 41-70.

Markman, E. M., \& Hutchinson, J. E. (1984). Children's sensitivity to constraints on word meaning: Taxonomic versus thematic relations. Cognitive Psychology, 16, 1-27.

Medin, D. L., \& WATTENMAKER, W. D. (1987). Category cohesiveness, theories, and cognitive archeology. In U. Neisser (Ed.), Concepts and conceptual development: Ecological and intellectual factors in categorization (pp. 25-62). Cambridge: Cambridge University Press.

Murphy, G. L., \& Allopenna, P. D. (1994). The locus of knowledge effects in concept learning. Journal of Experimental Psychology: Learning, Memory, \& Cognition, 20, 904-919.

Murphy, G. L., \& Medin, D. L. (1985). The role of theories in conceptual coherence. Psychological Review, 92, 289-316.

Newell, B. R., \& BRIGHT, J. E. H. (2001). The relationship between the structural mere exposure effect and the implicit learning process. Quarterly Journal of Experimental Psychology, 54A, 1087-1104.

NosofSKY, R. M. (1991). Tests of an exemplar model for relating perceptual classification and recognition memory. Journal of Experimental Psychology: Human Perception \& Performance, 17, 3-27.

PAZZANI, M. J. (1991). Influence of prior knowledge on concept acquisition: Experimental and computational results. Journal of Experimental Psychology: Learning, Memory, \& Cognition, 17, 416-432.

Perruchet, P., \& PaCteau, C. (1990). Synthetic grammar learning: Implicit rule abstraction or explicit fragmentary knowledge? Journal of Experimental Psychology: General, 119, 264-275.

Pothos, E. M. (in press). The rules versus similarity distinction. Behavioral \& Brain Sciences.

Pothos, E. M., \& BaILey, T. M. (2000). The role of similarity in artificial grammar learning. Journal of Experimental Psychology: Learning, Memory, \& Cognition, 26, 847-862.

Pothos, E. M., \& Chater, N. (1998). Generality of the abstraction mechanisms in artificial grammar learning. In M. A. Gernsbacher \& S. J. Derry (Eds.), Proceedings of the 20th Annual Conference of the Cognitive Science Society (pp. 854-858). Mahwah, NJ: Erlbaum.

Pothos, E. M., Chater, N., \& Stewart, A. J. (2004). Information about the logical structure of a category affects generalization. British Journal of Psychology, 95, 371-386.

Rathus, J. H., Reber, A. S., Manza, L., \& Kushner, M. (1994). Implicit and explicit learning: Differential effects of affective states. Perceptual \& Motor Skills, 79, 163-184.

REBER, A. S. (1967). Implicit learning of artificial grammars. Journal of Verbal Learning \& Verbal Behavior, 6, 855-863.

REBER, A. S. (1989). Implicit learning and tacit knowledge. Journal of Experimental Psychology: General, 118, 219-235.

REBER, A. S., \& ALLEN, R. (1978). Analogic and abstraction strategies in synthetic grammar learning: A functionalist interpretation. $\mathrm{Cog}_{-}$ nition, 6, 189-221.

Reber, A. S., Kassin, S., Lewis, S., \& Cantor, G. (1980). On the relationship between implicit and explicit modes in the learning of a complex rules structure. Journal of Experimental Psychology: Human Learning \& Memory, 6, 492-502. 
Reber, A. S., \& LewIS, S. (1977). Implicit learning: An analysis of the form and structure of a body of tacit knowledge. Cognition, 5, 333-361.

Reber, R., \& Perruchet, P. (2003). The use of control groups in artificial grammar learning. Quarterly Journal of Experimental Psychology, 56A, 97-115.

SHANKS, D. R., JOHNSTONE, T., \& STAGgS, L. (1997). Abstraction processes in artificial grammar learning. Quarterly Journal of Experimental Psychology, 50A, 216-252.

SHEPARD, R. N. (1980). Multidimensional scaling, tree-fitting, and clustering. Science, 210, 390-398.

TIPPER, S. P., \& CRANSTON, M. (1985). Selective attention and priming: Inhibitory and facilitatory effects of ignored primes. Quarterly Journal of Experimental Psychology, 37A, 591-611.

TuNNEY, R. J., \& SHANKS, D. R. (2003). Subjective measures of awareness and implicit cognition. Memory \& Cognition, 31, 1060-1071.

Wattenmaker, W. D. (1995). Knowledge structures and linear separability: Integrating information in object and social categorization. Cognitive Psychology, 28, 274-328.

WATTENMAKER, W. D. (1999). The influence of prior knowledge in in- tentional versus incidental concept learning. Memory \& Cognition, 27, 685-698.

WATtenmaKer, W. D., Dewey, G. L., Murphy, T. D., \& Medin, D. L. (1986). Linear separability and concept learning: Context, relational properties, and concept naturalness. Cognitive Psychology, 18, 158194.

Whittlesea, B. W. A., \& Wright, R. L. (1997). Implicit (and explicit) learning: Acting adaptively without knowing the consequences. Journal of Experimental Psychology: Learning, Memory, \& Cognition, 23, 181-200.

WISNIEWSKI, E. J. (1995). Prior knowledge and functionally relevant features in concept learning. Journal of Experimental Psychology: Learning, Memory, \& Cognition, 21, 449-468.

\section{NOTE}

1. For the purposes of the present work, this broad definition of implicit knowledge will suffice, so more specific approaches to understanding implicit knowledge (e.g., as unconscious) will not be considered.

\section{APPENDIX A}

The Frequency of Bigrams in the Training and Test Items of the A. S. Reber and Allen (1978) Letter String AGL Stimuli

\begin{tabular}{|c|c|c|c|c|c|c|c|}
\hline \multicolumn{2}{|c|}{ Training Items } & \multicolumn{2}{|c|}{ G Test Items } & \multicolumn{4}{|c|}{ NG Test Items } \\
\hline $\mathrm{MS}$ & 5 & $\mathrm{MS}$ & 8 & $\mathrm{MM}$ & 1 & SV & 6 \\
\hline MV & 5 & MV & 5 & MR & 1 & SX & 2 \\
\hline $\mathrm{RM}$ & 1 & $\mathrm{RM}$ & 3 & MS & 6 & VM & 1 \\
\hline RR & 6 & RR & 4 & MV & 2 & VR & 7 \\
\hline RX & 10 & $\mathrm{RX}$ & 10 & MX & 2 & VS & 5 \\
\hline SS & 5 & SS & 9 & $\mathrm{RM}$ & 3 & VV & 2 \\
\hline SV & 9 & SV & 11 & RR & 7 & VX & 7 \\
\hline VR & 10 & VR & 10 & $\mathrm{RS}$ & 4 & $\mathrm{XM}$ & 2 \\
\hline VS & 5 & VS & 5 & RV & 5 & XR & 7 \\
\hline VX & 10 & VX & 12 & $\mathrm{RX}$ & 6 & XS & 1 \\
\hline XM & 2 & XM & 1 & SM & 1 & XV & 7 \\
\hline XR & 4 & XR & 8 & SR & 5 & XX & 1 \\
\hline XS & 4 & XS & 3 & SS & 6 & & \\
\hline XV & 6 & XV & 8 & & & & \\
\hline
\end{tabular}

APPENDIX B

An Assignment of 0, 1, or 2 to Each Bigram That Reflects the Relative Length of Distances Between Letters According to the Figure 2 Arrangement

\begin{tabular}{llllllll}
\hline MM & 0 & RS & 2 & SV & 1 & VX & 0 \\
MR & 2 & RV & 0 & SX & 1 & XM & 2 \\
MS & 1 & RX & 0 & VM & 1 & XR & 0 \\
MV & 1 & SM & 1 & VR & 0 & XS & 1 \\
MX & 2 & SR & 2 & VS & 1 & XV & 0 \\
RM & 2 & SS & 0 & VV & 0 & XX & 0 \\
RR & 0 & & & & & & \\
\hline
\end{tabular}


APPENDIX C

Overall Distance Corresponding to Each of the NG and G Items of the A. S. Reber and Allen (1978) Stimuli

\begin{tabular}{|c|c|c|c|c|c|c|c|}
\hline \multicolumn{4}{|c|}{ NG Items } & \multicolumn{4}{|c|}{ G Items } \\
\hline MMVRX & 1 & SVSSXV & 3 & MSSSV & 2 & VXR & $\overline{0}$ \\
\hline MSM & 2 & SXRRM & 3 & MSSSVS & 3 & VXRM & 2 \\
\hline MSRV & 3 & VRRRM & 2 & MSSV & 2 & VXRRR & 0 \\
\hline MSRVRX & 3 & VVXRM & 2 & MSSVRX & 2 & VXRRRM & 2 \\
\hline MSSVSR & 5 & VXMRXV & 4 & MSV & 2 & VXSSSV & 2 \\
\hline MSVRSR & 6 & VXRRS & 2 & MSVRXR & 2 & VXSSV & 2 \\
\hline MSVV & 2 & VXRS & 2 & MSVRXV & 2 & VXSV & 2 \\
\hline MVRSR & 5 & VXRVM & 1 & MSVS & 3 & VXV & 0 \\
\hline MXVRXM & 4 & VXX & 0 & MVRXM & 3 & VXVRX & 0 \\
\hline MXVS & 3 & XRVXV & 0 & MVRXR & 1 & VXVRXR & 0 \\
\hline RRRXV & 0 & XSSSSV & 2 & MVRXRM & 3 & VXVRXV & 0 \\
\hline RVS & 1 & XVRXRR & 0 & MVRXVS & 2 & VXVS & 1 \\
\hline SSVS & 2 & & & MVS & 2 & & \\
\hline
\end{tabular}

Note-Overall distance was computed in terms of the association of each letter pair with a 0,1 , or 2 , shown in Appendix B.

(Manuscript received November 3, 2003;

revision accepted for publication May 7, 2004.) 